\title{
Model-Based Error Correction for Flexible Robotic Surgical Instruments
}

\author{
Ryan A. Beasley Robert D. Howe \\ Division of Engineering and Applied Sciences \\ Harvard University
}

\begin{abstract}
Robots promise to enhance minimally-invasive surgery, but flexion of the thin instrument shaft introduces error into models of the robot kinematics. Visual or electromagnetic tracking of the instrument tip provides correct forward kinematics, but uncertainty in shaft bending and port location leaves residual errors in inverse kinematics. These errors can cause incorrect motions that preclude the use of image-guidance tools. This paper proposes a model-based controller to correct the commanded motions. Comparison with a controller assuming a straight instrument shaft quantifies motion errors resulting from the use of a straight shaft controller. Analysis of the flexed shaft controller shows sensitivity to shaft length, shaft stiffness, tip force, and sensor noise.
\end{abstract}

\section{INTRODUCTION}

Image-guided robotic surgery uses three-dimensional medical image data to guide instruments to specific tissue locations identified in the image. This approach can involve either autonomous robotic control or human-in-the-loop techniques such as augmented reality and virtual fixtures $[1,2]$. In either case, good performance depends on accurately placing the instrument tip at the specified image coordinates.

Unfortunately, instrument shaft flexibility can prove a serious limitation. For example, a $1 \mathrm{~N}$ force applied to the tip of a cantilevered instrument for a commercial surgical robot (straight endoscissors for the ZEUS Surgical Robot System, Computer Motion, Inc, Goleta, Calif.; stainless steel shaft 355 $\mathrm{mm}$ long, $3.35 \mathrm{~mm}$ outer diameter, $1.47 \mathrm{~mm}$ inner diameter) causes a $15 \mathrm{~mm}$ tip deflection, much larger than the target anatomy in many procedures. Such bending has been shown to limit accuracy in animal studies of image-guided cardiothoracic procedures $[2,6]$. As surgeons seek to minimize instrument size to reduce damage to healthy tissue and access smaller anatomical structures, this problem will become more pronounced.

In terms of robot control, instrument flexibility alters the kinematic structure of the manipulator so that the assumed relationship between the robot's joint and tip coordinates becomes inaccurate. A number of approaches to kinematic error correction have been developed in the robotics literature. Offline calibration attempts to precisely determine kinematic parameters, but does not account for online changes resulting from interactions with unmodeled environments [7, 8]. Research in flexible robot manipulators handles changes in endpoint-joint relationships during operation, but focuses on dynamic flexibility $[9,10]$. Such an approach assumes known static kinematics, which is not the case for the quasi-static deformation seen in minimally invasive surgery.

Tracking the instrument tip with computer vision techniques or an electromagnetic sensor solves the problem of error in the forward kinematics, as the instrument tip coordinates are directly measured. This measurement enables the use of image guidance in robotic minimally invasive procedures. Measuring the tip position will not directly correct the inverse kinematics, unfortunately. The inverse kinematics will still be modeled off a straight instrument shaft, and can cause divergent motions of the instrument tip even with tip tracking [11]. With tip measurements and known errors in the kinematic model, there are convergence metrics for the error in Jacobian-based controllers, but these metrics do not reduce the motion errors $[11,12]$.

A model-based controller is a logical approach to reducing flexion-induced errors. In this paper we develop a mechanical model of the instrument shaft deflection, based on a simply supported beam. Using the developed model and tip tracking, we propose a real-time method to reduce the errors in the control of a surgical instrument. The metric introduced in [11] is used to quantify the convergence properties of the resulting Jacobian controller in the presence of measurement error. Simulations compare the resulting controller with a controller assuming a rigid instrument shaft. Sensitivity analysis shows the effect of measurement noise on the proposed controller.

\section{CONTROller Design}

\section{A. Flexion Model}

Fig. 1 diagrams a robot-assisted surgical procedure in which the instrument shaft is flexing. For the purposes of the following analysis, the instrument shaft is modeled as a straight rod, connected to the robot arm at a wrist. The controller's job is to accurately position the tip in a world coordinate frame. As in many laparoscopic and thoracoscopic procedures, we assume that the instrument is introduced into the patient's body through an endoscopic port, a rigid cannula a few $\mathrm{cm}$ in length that is tightly inserted into a small incision. As the instrument tip interacts with tissue, radial tip forces generate reaction forces at the port and at the robot wrist. These forces result in bending of the instrument.

The goal is then to determine the actual kinematics of the instrument, i.e. its shape as it flexes during the procedure. 


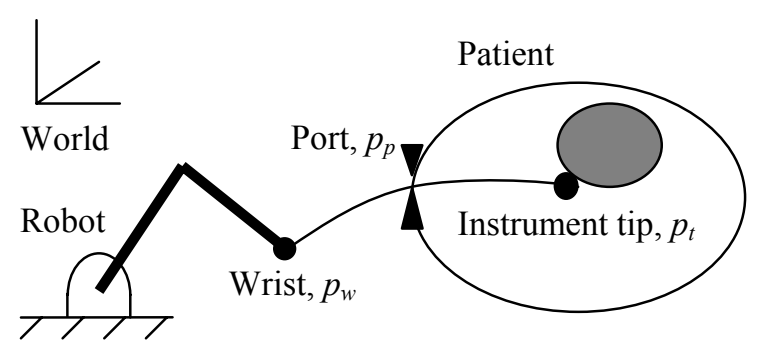

Fig. 1. Flexion in surgical instrument shaft due to force on tip.

The Euler-Bernoulli beam bending equations were chosen to model the instrument flexion, with an assumption of a simply supported configuration, i.e., the wrist, port, and tip apply only forces normal to the shaft and no moments (Fig. 2). The simply supported configuration fits the situation where just the tip of the instrument is in contact with the patient and this model precludes complicated shapes such as s-curves in the interest of low complexity. With both ends of the beam on the $\mathrm{x}$-axis the height of the beam in model space ([14]) is

$$
y_{m}\left(x_{m}\right)=\frac{-F_{p}}{6 E I}\left(L b x_{m}\left(1-\frac{b^{2}}{L^{2}}-\frac{x_{m}^{2}}{L^{2}}\right)+\left\langle x_{m}-a\right\rangle^{3}\right)
$$

and the slope of the beam is

$$
y_{m}^{\prime}\left(x_{m}\right)=\frac{-F_{p}}{6 E I}\left(L b\left(1-\frac{b^{2}}{L^{2}}-\frac{3 x_{m}^{2}}{L^{2}}\right)+3\left\langle x_{m}-a\right\rangle^{2}\right)
$$

where $x_{m}$ is the position along the beam, $F_{p}$ is the force at the port acting at a distance $a$ along the beam from the wrist, $L$ is the total length of the beam, $b=L-a, E$ is the Young's modulus, $I$ is the cross sectional moment of inertia, and

$$
\left\langle x_{m}-a\right\rangle^{1}=\left\{\begin{array}{cc}
0 & x_{m} \leq a \\
\left(x_{m}-a\right) & x_{m} \geq a
\end{array} .\right.
$$

$F_{p}$ and $a$ are the two unknowns in this model, so two measurements of the beam position, slope, or curvature are necessary for a solution. Additionally, measurements are necessary for registration between world space and model space.

We assume that instrument positions and orientations (slopes) are sensed near both ends: at the tip end using either electromagnetic or visual sensing, and at the wrist end using either electromagnetic or robot arm kinematic sensors (e.g., high resolution joint encoders). The measurements permit

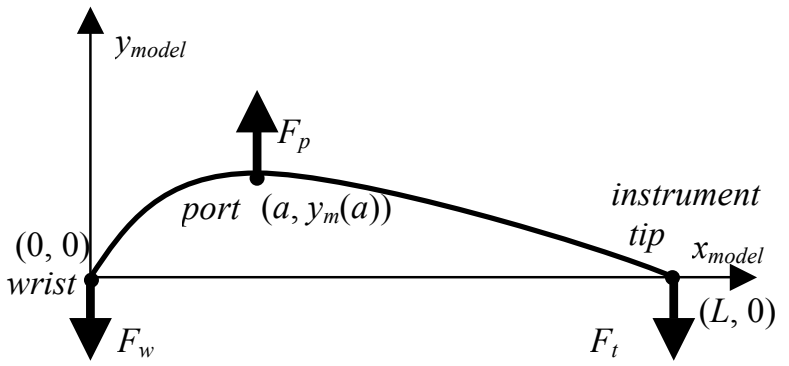

Fig. 2. Simply supported beam model of instrument shaft in model space.

calculation of the model parameters, and thus the Jacobian relating the motion of the beam's endpoints. Similarly, the port position or wrist force, port force, or tip force could be measured directly instead of the sensors used here; these alternatives are evaluated in the Discussion section below.

\section{B. Jacobian Construction}

Fig. 3 diagrams the inverse-Jacobian controller used in this paper and described in [11]. The premise is that the existing joint level controller accepts a desired angular configuration

$$
\theta_{d}=\theta+\left(J_{i} J_{r}\right)^{-1}\left(p_{\text {desired }}-p_{t}\right)
$$

where $\theta$ is the vector of current joint angles, $J_{r}$ is the robot Jacobian relating joint motions to wrist motions, $J_{i}$ is the Jacobian relating motions of the wrist end of the instrument shaft to motions of the tip end, $p_{\text {desired }}$ is the desired tip position, and $p_{t}$ is the current tip position. The appeal of Jacobian-based controllers is that they move the instrument tip directly towards the desired position in world space, with clear safety advantages for working inside a patient's body. Dynamics are unnecessary because surgical robots move slowly, are highly geared, and are often in contact with viscoelastic tissues, resulting in a quasi-static system. We assume that the robot arm can precisely position the wrist and the robot's Jacobian is known, so our task is to find the Jacobian for the instrument shaft. For clarity, we consider position control in 2-D here; orientation control and extension to 3-D are discussed below.

The Jacobian of the instrument shaft is constructed by taking the partial derivative of the tip position with respect to the robot wrist position

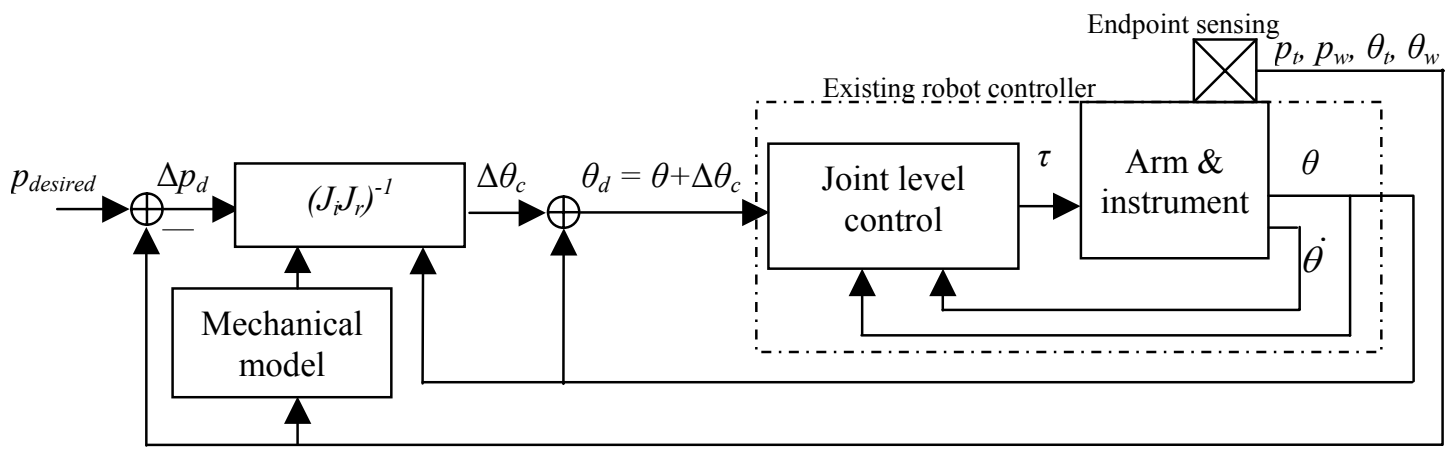

Figure 3. Block diagram of controller. 


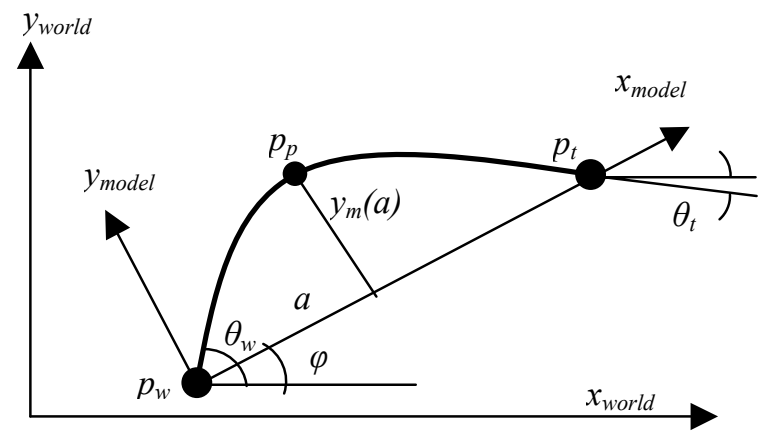

Fig. 4. Relationship between world and model frames

$$
J_{i}=\left[\begin{array}{ll}
\frac{\partial x_{t}}{\partial x_{w}} & \frac{\partial x_{t}}{\partial y_{w}} \\
\frac{\partial y_{t}}{\partial x_{w}} & \frac{\partial y_{t}}{\partial y_{w}}
\end{array}\right]
$$

where $p_{w}=\left(x_{w}, y_{w}\right)$ is the robot wrist position in world space, and $p_{t}=\left(x_{t}, y_{t}\right)$ is the instrument tip position in world space. The position of the instrument tip in world space can be written as a transformation from model space

$$
\left[\begin{array}{l}
x_{t} \\
y_{t}
\end{array}\right]=L\left[\begin{array}{l}
\cos (\varphi) \\
\sin (\varphi)
\end{array}\right]+\left[\begin{array}{l}
x_{w} \\
y_{w}
\end{array}\right]
$$

where $\varphi$ is the angle between model space and world space (Fig. 4). To incorporate the port location, $p_{p}$, which determines the kinematic relationship between the wrist and tip, this angle can be written as

$$
\varphi=\operatorname{Tan}^{-1}\left(\frac{y_{p}-y_{w}}{x_{p}-x_{w}}\right)-\operatorname{Tan}^{-1}\left(\frac{y_{m}(a)}{a}\right) .
$$

Combining (1), (5), (6), and (7), and performing the derivative operations in (5), the Jacobian can be written as a function of $p_{w}, p_{p}, a, F_{p}, y_{m}(a)$, and known parameters. Now the terms $p_{p}, a, F_{p}$, and $y_{m}(a)$ must be written as functions of the position and angle measurements at the instrument endpoints, $p_{w}, p_{t}, \theta_{w}$, and $\theta_{t}$. The port position in world space can be written as a transformation from its position in model space

$$
\begin{gathered}
{\left[\begin{array}{l}
x_{p} \\
y_{p}
\end{array}\right]=\left[\begin{array}{cc}
\operatorname{Cos}(\varphi) & -\operatorname{Sin}(\varphi) \\
\operatorname{Sin}(\varphi) & \operatorname{Cos}(\varphi)
\end{array}\right]\left[\begin{array}{c}
a \\
y_{m}(a)
\end{array}\right]+\left[\begin{array}{l}
x_{w} \\
y_{w}
\end{array}\right]} \\
\varphi=\operatorname{Tan}^{-1}\left(\frac{y_{t}-y_{w}}{x_{t}-x_{w}}\right) .
\end{gathered}
$$

From (1), the deflection height at the center force can be written in terms of $F_{p}$ and $a$

$$
y_{m}(a)=-\left(\frac{F_{p}}{E I}\right)\left(\frac{a^{2}(a-L)^{2}}{3 L}\right) .
$$

$F_{p}$ and $a$ can be written as functions of the orientation measurements

$$
F_{p}=\frac{2 E I\left(y_{m}{ }^{\prime}(0)-y_{m}{ }^{\prime}(L)\right)}{a(a-L)}
$$

$$
a=\frac{L\left(2 y_{m}{ }^{\prime}(L)+y_{m}{ }^{\prime}(0)\right)}{y_{m}{ }^{\prime}(L)-y_{m}{ }^{\prime}(0)}
$$

where

$$
\begin{aligned}
& y_{m}^{\prime}(0)=\operatorname{Tan}\left(\theta_{w}-\varphi\right) \\
& y_{m}^{\prime}(L)=\operatorname{Tan}\left(\theta_{t}-\varphi\right) .
\end{aligned}
$$

Combining these equations permits calculation of the desired form of the Jacobian, which is reproduced in the appendix.

\section{EVALUATION}

To quantify the error in this inverse Jacobian controller we use a metric from [11]. The true and estimated Jacobians, $J_{i}$ and $\hat{J}_{i}$, are combined to create a map from the current position error to the position error after a motion commanded by the estimated Jacobian (Fig. 5)

$$
G=I-J_{i} \hat{J}_{i}^{-1} .
$$

The induced Euclidean norm then finds the worst case for the resulting ratio between the new and old error lengths

$$
\|G\|_{i 2}=\max _{\Delta p_{d} \neq 0} \frac{\left\|G \Delta p_{d}\right\|_{2}}{\left\|\Delta p_{d}\right\|_{2}}=\bar{\sigma}(G)
$$

where $\Delta \mathrm{p}_{d}$ is the old error vector.

If this metric is less than 1 across the workspace, then commanded motions always shrink the length of the position error. Therefore, values below 1 indicate monotonic position convergence (lower values signifying faster convergence), and values above 1 indicate the potential for divergence (higher values signifying faster divergence).

\section{METHODS}

The metric explained in the previous section will be used to compare the proposed controller with a controller based on kinematics of a straight shaft. The metric will also be used to determine the sensitivity of the proposed controller to sensor noise.

\section{A. Comparison with a Rigid Instrument Model}

Using the proposed metric, the controller introduced in this work can be compared with other controllers. The controller for the Zeus Surgical System assumes a rigid instrument shaft

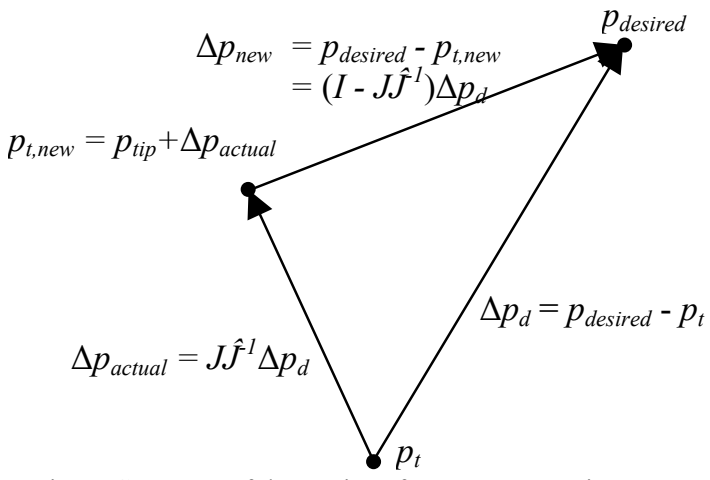

Fig. 5. Geometry of the motions for an erroneous inverse Jacobian controller. 


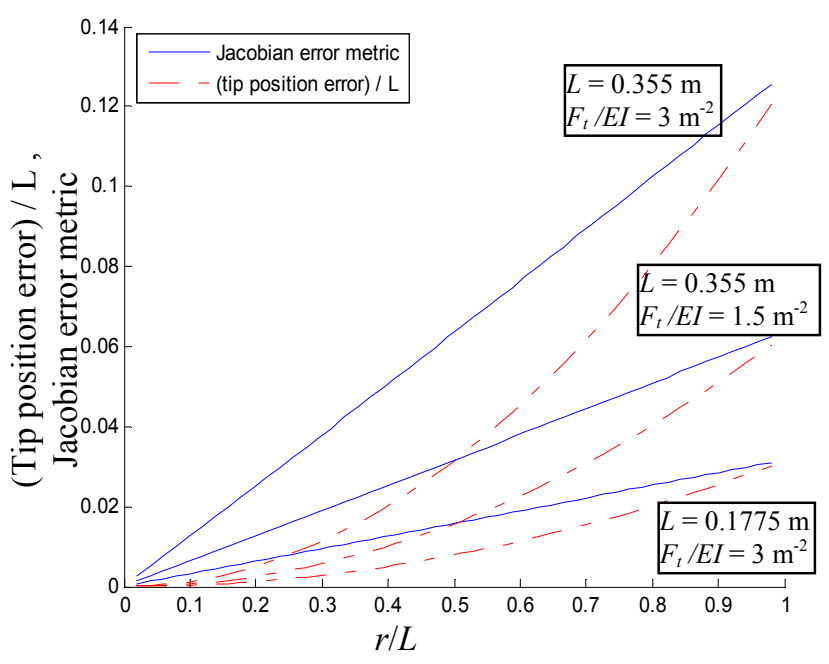

Fig. 6. Position error and Jacobian motion increase as the instrument tip is inserted farther into the port.

and uses a Jacobian based on the lever equation

$$
\left[\begin{array}{l}
x_{t} \\
y_{t}
\end{array}\right]=\frac{L}{\left\|p_{p}-p_{w}\right\|}\left[\begin{array}{l}
x_{p}-x_{w} \\
y_{p}-y_{w}
\end{array}\right] .
$$

Without direct measurement, the port position, $p_{p}$, is estimated over successive controller cycles. The port position is chosen as the intersection point of rays drawn along the instrument shaft as measured by wrist sensors. To quantify the benefits of tip tracking, the distance can be calculated between the actual tip position and the tip position estimated by the current Zeus rigid lever controller. To calculate the actual tip position, first the right triangle formed by $a$ and $y_{m}(a)$ is solved for $a$ (Fig. 4)

$$
\left\|p_{p}-p_{w}\right\|^{2}=a^{2}+y_{m}^{2}(a)
$$

with (1) substituted in for $y_{m}(a)$. Then (1), (6), and (7) are combined to calculate the tip position. In addition, to quantify the benefits of the flexion model, the induced Euclidean norm can be used to compare the lever Jacobian with the flexion Jacobian. The rigid instrument Jacobian is calculated by substituting (17) into (5).

\section{B. Sensitivity Analysis for Jacobian}

To compute the effects of sensor noise on the proposed controller, the induced Euclidean norm is used again. The flexion model Jacobian is substituted into (15) as both the true and estimated Jacobians, the latter instance being calculated with additive sensor noise. The noise has a uniform distribution along each sensor axis, with a maximum equal to the number listed in the figures and a minimum equal to the negative of the same number. To investigate convergence, the induced Euclidean norm is then evaluated at various insertion depths and with various magnitudes of sensor noise. For a given insertion depth, the maximum error across noise values is shown. One of the advantages of the flexion model is an estimate of the port position, the accuracy for which is unaffected by flexion. Error in port localization due to sensor noise is also calculated.

\section{RESULtS}

For all plots, the tip and wrist forces will be assumed to act such that $F_{w}$ and $F_{t}$ are in the $-y_{m}$ direction in model space; axial forces in the $x_{m}$ direction do not produce bending. Taking advantage of rotational symmetry about the port, all plots show the values as a function of insertion depth, $r=\left[\left(x_{t}\right.\right.$ $\left.\left.-x_{p}\right)^{2}+\left(y_{t}-y_{p}\right)^{2}\right]^{1 / 2}$.

\section{A. Comparison with a Rigid Instrument Model}

The greater the insertion depth, the larger the error in tip position for the current Zeus controller without tip tracking (Fig. 6). Additionally, the motion error for the rigid instrument Jacobian is proportional to the insertion depth, as determined by (16) with the flexion model Jacobian as the true Jacobian.

\section{B. Sensitivity Analysis for Jacobian}

Noise in the position and orientation measurements degrades the performance of the flexion model Jacobian. To demonstrate these effects, (16) was calculated across the tip workspace, using the flexion Jacobian with noise in each measurement for the estimated Jacobian and without measurement noise for the true Jacobian. The following plots show the worst (maximum) values across all noise directions.

In the presence of sensor noise, the motion errors from the flexion model Jacobian are smallest when the instrument is inserted approximately $70 \%$ of its length (Fig. 7). The motions become divergent as either the tip or the wrist move close to the port. Decreasing the sensor noise reduces the controller's motion errors.

The estimated parameters, $F_{p}$ and $a$, can also be examined. Since $F_{p}$ and the structural rigidity, $E I$, always enter the beam bending equations together, the term $F_{p} / E I$ was estimated. This term can be thought of as a normalized force. $F_{p} / E I$ can be incorrect by orders of magnitude outside of the convergent area due to the measurement noise, but improves as $r / L$ approaches 0.5 (Fig. 8). The error in estimating the port position decreases as the instrument tip moves further from the port (Fig. 9). Increasing the instrument length, $L$, or the normalized force, $F_{t} / E I$, increases the convergence of the controller, similar to decreasing sensor noise (Fig. 10). Increasing these parameters increases flexion for a constant tip force. For a given level of sensor noise, increasing the flexion would increase the signal-to-noise ratio, explaining the similarity between Figs. 7 and 10 .

\section{DISCUSSION}

Tracking the instrument tip in minimally invasive surgery avoids errors in the forward kinematics and allows a more accurate kinematic model for motion control. The modelbased controller presented here for flexible instrument shafts can reduce motion error in comparison with controllers assuming rigid links. The simply supported beam models the 


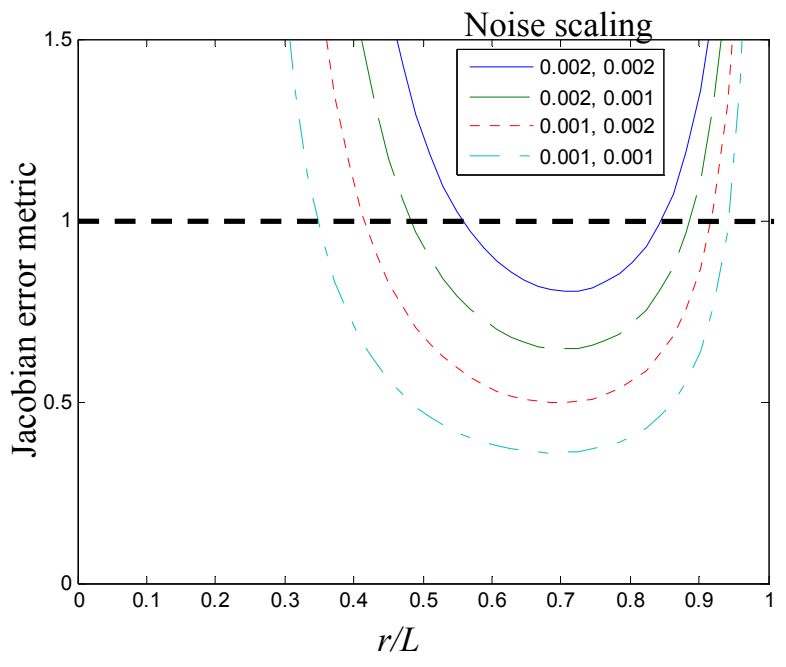

Fig. 7. The motions commanded by the flexion based Jacobian become less accurate as sensor noise increases. Values greater than 1 signify divergent motions. The first scaling number is the position error scaling, with respect to $L$. The second number is the angular error (rad). $L=0.355 \mathrm{~m} . F_{t} / E I=3 \mathrm{~m}^{-2}$.

situation in which the only forces on the instrument are at the wrist, port, and tip, and there are no moments. Barring sensor noise, the flexion model commands accurate motions in this situation whereas the rigid instrument model results in motion errors.

To compare the sensor noise magnitudes used in this paper to a commercial product, the published RMS accuracy of the Liberty magnetic tracking system is $0.76 \mathrm{~mm}$ position and 0.15 degrees orientation or about $0.0021 L$ and 0.0026 radians (Polhemus Technologies, Colchester, VT). For this level of noise with $L=0.355 \mathrm{~m}$ and $F_{t} / E I=3 \mathrm{~m}^{-2}$, the insertion depth should be constrained to $0.55<r / L<0.85$ to ensure monotonically convergent motions (Fig. 7). Improved signal processing or a tracking technology with less noise would relax these bounds on insertion depth.

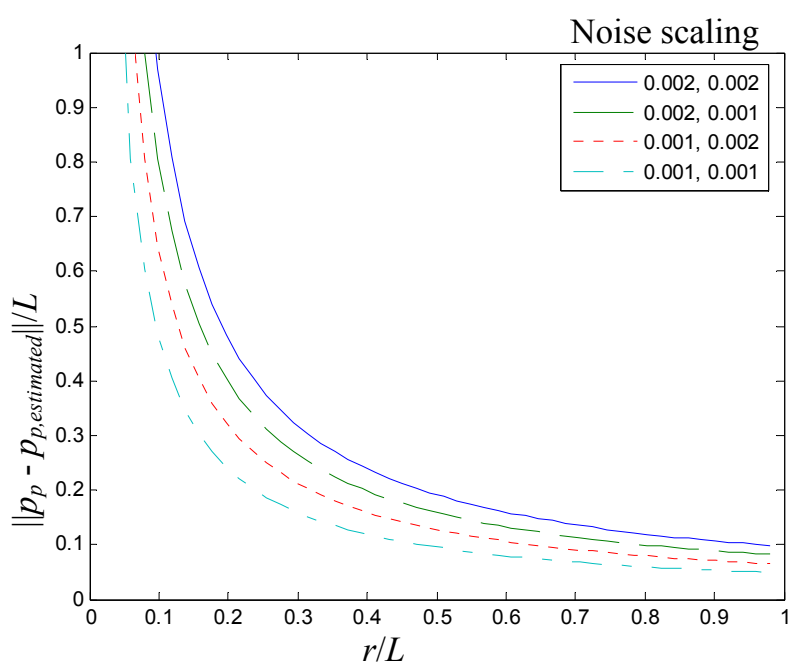

Fig. 9. The error in port position estimation decreases as insertion depth increases. The first scaling number is the position error scaling, with respect to $L$. The second number is the angular error (rad). $L=$ $0.355 \mathrm{~m} . F_{t} / E I=3 \mathrm{~m}^{-2}$.

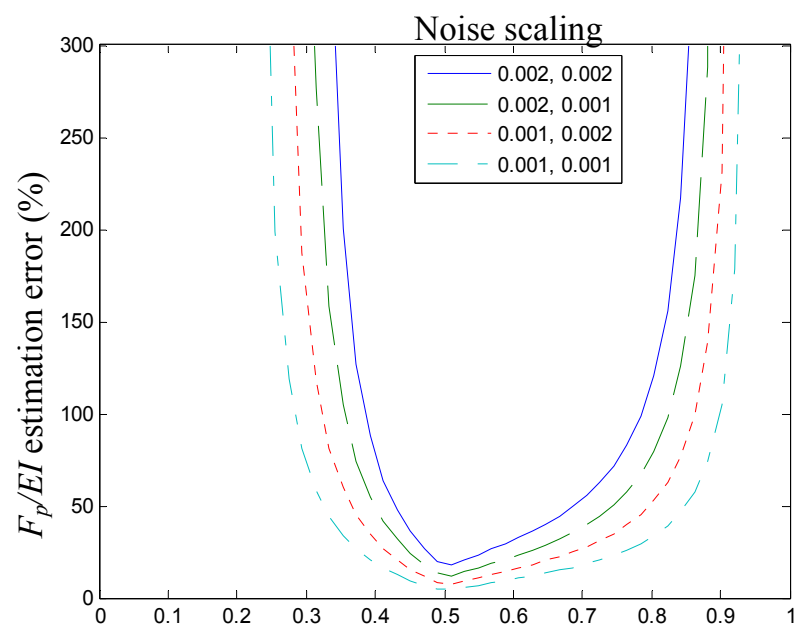

Fig. 8. In the presence of sensor $r$ noise, $F_{p} / E I$ is best estimated in the center of the workspace. The first scaling number is the position error scaling, with respect to $L$. The second number is the angular error (rad). $L=0.355 \mathrm{~m} . F_{t} / E I=3 \mathrm{~m}^{-2}$.

As shown in Fig. 6, the error in tip position estimation can be on the order of centimeters if a bent instrument shaft is assumed to be straight. Such an error magnitude is larger than the sizes of many surgical targets $[2,6]$. Tip tracking measures the actual position of the tip, allowing effective image guidance.

In this paper, the positions and orientations of the instrument's endpoints are used to solve for the flexion model parameters. Other measurement possibilities exist, however, including the port position and orientation, and forces/torques on the wrist, port, and tip. Of these possibilities, measuring the force and torque at the robot wrist is the most feasible. Accurately measuring the position and orientation of the instrument shaft at the port is difficult due to the uncertain relationship between the physical port and any contact points between the port and the instrument shaft. Similarly, the difficulty of measuring the forces exerted on the port, or the

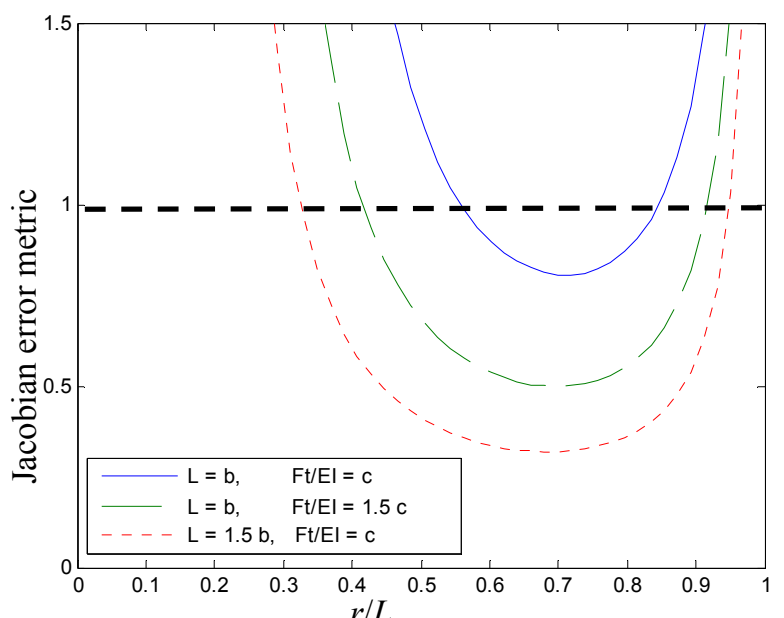

Fig. 10. The motions commanded by the flexion based Jacobian become more accurate as either $L$ increases or the tip force increases. $b=0.355 \mathrm{~m} . c=3 \mathrm{~m}^{-2}$. The noise magnitude is $0.002 \mathrm{~L}$ for the position measurements and $0.002 \mathrm{rad}$ for the angle measurements. Values greater than 1 signify divergent motions. 
instrument tip, obstruct direct measurement of one of the model parameters, $F_{p}$. Construction of a force sensor small enough to fit through the port, or of a port incorporating a force sensor, would allow this direct measurement.

The Jacobian in this paper only handles motions in the plane containing the instrument, but is easily extended to 3-D. If the robot exerts a force on the instrument that is perpendicular to the plane of bending, torque will be generated at the port position. The entire plane of bending will then rotate about a line that passes through the port position.

Orientation control can be achieved by calculating the Jacobian relating the change of orientation of the tip to the change in position of the wrist. Assuming infinitesimal motions, moving the wrist does not change the shape of the beam, so the only change in orientation is caused by the changing registration between the model and world spaces. The columns of the positional Jacobian matrix describe the tip motions resulting from wrist motions along the world space axes, allowing calculation of the consequential change in rotation.

In the interests of a better fit between the model and the true instrument shaft shape, the simply supported configuration can be replaced with a more complicated assumption of the shape. The new configuration would allow the controller to handle more complex instrument/environment interactions, but would contain more unknowns and thus require correspondingly more measurements. Those measurements could come from a force/torque sensor as discussed above or from additional position and orientation measurements.

\section{APPENDIX: FLEXION JACOBIAN}

$$
\begin{gathered}
J_{i}=\left[\begin{array}{ll}
J_{1,1} & J_{1,2} \\
J_{2,1} & J_{2,2}
\end{array}\right] \\
J_{1,1}=1+L \operatorname{Sin}(\gamma-\varphi) \\
\left(\frac{\Delta Y}{L_{\text {meas }}^{2}}+\frac{\Delta X(\text { temp } 2-\text { temp } 1)}{\text { temp } 3}\right) \\
J_{1,2}=L \operatorname{Sin}(\gamma-\varphi) \\
\left(\frac{-\Delta X}{L_{\text {meas }}^{2}}+\frac{\Delta Y(\text { temp } 2-\text { temp } 1)}{\text { temp } 3}\right) \\
J_{2,1}=-L \operatorname{Cos}(\gamma-\varphi) \\
\left(\frac{-\Delta Y}{L_{\text {meas }}^{2}}+\frac{\Delta X(\text { temp } 1-\text { temp } 2)}{\text { temp } 3}\right) \\
J_{2,2}=1-L \operatorname{Cos}(\gamma-\varphi) \\
\left(\frac{\Delta X}{L_{\text {meas }}^{2}}+\frac{\Delta Y(\text { temp } 1-\text { temp } 2)}{\text { temp } 3}\right)
\end{gathered}
$$

$$
\begin{gathered}
\gamma=\operatorname{Tan}^{-1}\left(\frac{y_{m}(a)}{a}\right) \\
\text { temp } 1=3 L y_{m}(a) \\
\text { temp } 2=(2 a-L) \text { temp } 1 \\
\text { temp } 3=\left(3 L a+\text { temp } 2 * y_{m}(a)\right)\left(a^{2}+y_{m}(a)^{2}\right) \\
\Delta X=x_{p}-x_{w} \\
\Delta Y=y_{p}-y_{w} \\
L_{\text {meas }}=\left(\Delta X^{2}+\Delta Y^{2}\right)^{\frac{1}{2}}
\end{gathered}
$$

\section{REFERENCES}

[1] T. M. Peters, "Image-guided surgery: from X-rays to virtual reality," Computer Methods in Biomechanics and Biomedical Engineering, vol. 4, pp. 27-57, 2000.

[2] S. S. Park, R. D. Howe, and D. F. Torchiana, "Virtual fixtures for robotassisted minimally-invasive cardiac surgery," Medical Image Computing and Computer-Assisted Intervention 2001, W.J. Niessen and M.A. Viergever, Eds. Berlin: Springer-Verlag, Berlin, 2001, pp. 14191420.

[3] J. J. Abbott and A. M. Okamura, "Virtual fixture architectures for telemanipulation," IEEE Intl. Conf. on Robotics and Automation, pp. 2798-2805, 2003.

[4] G. Guthart and K. Salisbury, Jr., "The IntuitiveTM telesurgery system: overview and application," IEEE Intl. Conf. on Robotics and Automation, pp. 618-621, 2000.

[5] R. A. Beasley and R. D. Howe, "Tactile tracking of arteries in robotic surgery," IEEE Intl. Conf. on Robotics and Automation, Vol. 4, pp. 3801-3806, 2002.

[6] E. Coste-Maniere, L. Adhami, F. Mourgues, and O. Bantiche, "Optimal planning of robotically assisted heart surgery: first results on the transfer precision in the operating room," Intl. J. of Robotics Research, vol. 23, no. 4-5, pp. 539-48, 2004.

[7] J. M. Hollerbach and C. W. Wampler, "The calibration index and taxonomy for robot kinematic calibration methods," Intl. J. of Robotics Research, vol. 15, no. 6, pp. 573-591, 1996.

[8] Z. Roth, B. W. Mooring, B. Ravani, "An overview of robot calibration," IEEE Trans. Robotics and Automation, vol. 3, no 5, pp $377-385,1987$.

[9] D. M. Rovner and R. H. Cannon, Jr., "Experiments toward on-line identification and control of a very flexible one-link manipulator," Intl. J. of Robotics Research, vol. 6, no. 4, pp. 3-19, 1987

[10] D.-S. Kwon and W. J. Book, "A time-domain inverse dynamic tracking control of a single-link flexible manipulator," J. of Dynamic Systems, Measurement, and Control, vol. 116, pp. 193-200, 1994.

[11] R. A. Beasley, R. D. Howe, and P. E. Dupont. "Kinematic Error Correction for Minimally Invasive Surgical Robots," IEEE Intl. Conf. on Robotics and Automation, vol. 1, pp. 358-64, 2004.

[12] C. C. Cheah, M. Hirano, S. Kawamura, and S. Arimoto, "Approximate Jacobian control for robots with uncertain kinematics and dynamics," IEEE Trans. Robotics and Automation, vol. 19, no. 4, pp. 692-702, 2003.

[13] T. Belendez, C. Neipp, and A. Belendez, "Large and small deflections of a cantilever beam," Eur. J. Phys., vol. 23, pp 371-379, 2002.

[14] T. J. Lardner and R. R. Archer. Mechanics of solids: an introduction. New York: McGraw-Hill, 1994. 